\title{
Sports injuries: what is new today?
}

The outlook for complete recovery and rehabilitation from sports injuries has never been better

In recent years, the sports and leisure industry has become one of the fastest growing sectors of the business market, with a proportionate increase in sports injuries. An estimated 1.5 million people in Britain [1,4], and 3.7 million people in the USA [2] attend emergency departments each year, with an injury related to sports and exercise. In Asia too, though statistical data is not available, it is apparent that sports injuries are on the rise. As the number of sports injuries increased, the advances in management methodology have kept pace. Today, the outlook for an injured athlete is far more optimistic than in the past. Sports medicine has developed some near-miraculous ways to help athletes heal and hasten their return to sports. Despite this, many people, even today, think of sports medicine as merely treatment of athletic injuries and rehabilitation of sportspersons. In fact, the field of sports medicine has evolved to provide guidance on different aspects of sports related health, such as injury prevention, athletic conditioning, and proper sports equipment.

When it comes to sports, pain and gain often go hand-in-hand. Some sports related injuries are only temporary while others can cause lasting damage. The term sports injury, in the broadest sense, refers to the injuries that most commonly occur during sports or exercise. Some sports injuries result from accidents, others are due to poor training practices, improper equipment, lack of conditioning, or insufficient warm-up and stretching. Overall, sports injuries fall into two basic categories: overuse injuries and acute injuries. Although virtually any part of the body can be injured during sports or exercise, the term is usually reserved for injuries that involve the musculoskeletal system, which includes the muscles, bones, and associated tissues like cartilage. 
A better understanding of how sports injuries occur has significantly helped in reducing many of them. High speed video technology has allowed us to study the movements during a sports event; for example, a close watch at the motion of throwing a ball or tennis strokes, has helped us analyse where injuries occur and how best they can be prevented. The players can be counselled about their playing styles and the necessary modifications made if a particular action is hurting them, thus preventing injury. The common example is modifying a fast bowler's action to reduce incidence of stress fractures in lower lumbar spine. Screening for muscle imbalances is the current cutting edge of injury prevention. The rationale behind this is that there are detectable and correctable abnormalities of muscle strength and length that are fundamental to the development of almost all musculoskeletal pain and dysfunction. Similarly, structural abnormalities can place uneven stress on body parts (e.g. unequal leg length, pes-cavus, flat feet). The biomechanical factor that most commonly causes foot, leg, or hip injury is excessive pronation of the foot while running. Pronation helps prevent injury by distributing the force of impact with the ground; however, excessive pronation can cause injury through exaggerated medial twisting of the lower leg, resulting in foot, leg, hip, and knee pain. Also, patients with exaggerated lumbar lordosis are at risk of back pain when they swing a cricket or a baseball bat. Stress fractures can frequently be prevented with proper shoe wear, soft cushioned orthotics, and proper training techniques. Similarly, many muscle strains can also be prevented with proper warm-up and stretching prior to and post-activity.

For understanding these mechanics of injury, the concept of team doctors, who are sports medicine specialists, is the need of the hour in this part of the world. These specialists can critically and scientifically evaluate the players, before the match, during the match and during the off-season periods and advise the team management at every stage. These team physicians are closely associated with the team over a period of time. Their constant supervision helps in early diagnosis of any medical problem and its prompt treatment.

In previous years, the diagnosis of an injury was dependent on the clinical skills of the doctor, in addition to imaging techniques like xrays. However, newer diagnostic techniques such as CT and MRI scans, have aided greatly in early diagnosis and better treatment planning. Many studies have proved their efficacy and provided a high confidence interval in diagnosing occult lesions related to sporting activities.

Management protocols have also evolved; drug delivery has improved with the availability of pain-killing medicated patches that can be applied directly to the injury site. This effective method avoids the medication entering the general system and minimises drug attributable side-effects.

The advent of arthroscopic surgery has helped to revolutionise sports medicine treatment. In fact, fiberoptics of the arthroscope has become so sophisticated that the surgeon can usually see better inside the joint than could previously be seen through large incisions. Diagnostic ability has improved, and surgical access through minimally invasive methods allows anatomic tissue repair. Meniscal tears, that previously required removal, and would eventually lead to arthritic changes, now can frequently be repaired, preventing arthritic changes and improving function. Other advances in arthroscopy include the treatment of shoulder instability and rotator cuff problems. Previously shoulder pain meant either rotator cuff tendinitis or biceps pathology, but now with the aid of arthroscopy, we are able to visualise and delineate various shoulder pathologies like SLAP lesions, newer instability patterns and capsular laxity. Smaller instruments make joints (elbow, wrist, carpal tunnel, ankle, hip and the great toe) more amenable to arthroscopy. There is less trauma, swelling and scar tissue than conventional surgery, which in turn decreases the period of hospitalisation and rehabilitation. Furthermore, because injuries are often addressed at an early stage, operations are more likely to be successful and the athlete is able to return to play in quick time.

\section{The future}

Genetics is a branch that holds promise in sports medicine. Chronic traumatic brain injury, which occurs primarily among retired boxers (also known as dementia pugilistica, chronic traumatic encephalopathy, or "punchdrunk" syndrome), is an example of its application. The condition represents the cumulative long term neurologic consequence of repetitive concussive and subconcussive blows to the head. A milder form can occur in American football, ice hockey, rugby, soccer, or any sport associated with traumatic brain injury. Evidence suggests that apolipoprotein E epsilon-4 (apo E-e4), a susceptibility gene for late-onset familial and sporadic Alzheimer's disease, may also be associated with an increased risk of chronic traumatic brain injury in boxers [3]. Finding of this genetic predisposition to brain injury has implications not only for the medical regulation of boxing and other contact sports but also for our awareness of a need to understand better the interaction between genetic susceptibility and environmental triggers. With future advances we may be able to identify genes that predispose athletes to other sports related injuries. For example, we may be able to identify those who are at increased risk of rupture of the anterior cruciate ligament, multidirectional instability of the shoulder, etc.

Tissue engineering is another exciting development. Injured joint cartilage does not heal on its own the way other tissues do. In recent years, the transplantation of one's own healthy cartilage or cultured cells can help in healing sports related injuries. At present, this technique is used for small cartilage defects.

The concept of focused surgeons is here to stay [5]. But a team approach, in sports medicine centers, having 
all the specialists under one roof is the demand of the future. The orthopaedic super-specialists work cohesively, supported by internal medicine specialists, cardiologists, dermatologists, gynaecologists, physiologists and rehabilitation specialists. Such super-specialist care for the sportsperson would help in managing sports injuries in a professional manner. All surgeons have a learning curve and this is even steeper in case of arthroscopists. In this modern scenario, where focused surgeons limit themselves to one area of the body, they produce better results.

One of the greatest advances in sports medicine is rehabilitation after injuries. Physical therapy and occupational therapy programmes and protocols have been developed for virtually all sports related injuries. This allows many injuries to be effectively treated non-surgically and still allow them to heal while the athlete is participating in sports. The post-operative regimens allow many athletes to return to sports, weeks or months sooner, than they had done previously, prior to the rehabilitation advances. Improvements in sports equipment and technology have also helped to reduce injuries.

These recent advances in treating sports injuries are just the beginning. Newer research in the future will open new vistas. Technical advances in imaging methods will lead to better ways of diagnosing and treating injuries.
Researchers hope to gain a better understanding of the role of nutrition in inflammation and healing. The use of gene therapy may enable doctors to modify particular cells to induce repair of injured tissues, although ethical and legal issues of gene therapy are being debated. Whatever the future, we can safely say that sports medicine has evolved to an extent that it is now being considered a specialised branch of medicine.

\section{References}

1. Boyce SH, and Quigley MA. Review of sports injuries presenting to an accident and emergency department. Emergency Medicine Journal 2004;21:704-6.

2. Burt CW, Overpeck, MD. Emergency visits for sports-related injuries. Annals of Emergency Medicine 2001;37: 301-8.

3. Jordan BD. Genetic susceptibility to brain injury in sports: A role for genetic testing in athletes. The Physician and Sports Medicine 1998;26:25-6.

4. Nicholl JP, Coleman P, Williams BT. Pilot study of the epidemiology of sports injuries and exercise related morbidity. British Journal of Sports Medicine 1991;25:61-6

5. Dhillon MS, Nagi ON. Editorial : Trends in sports medicine. Bulletin of the Postgraduate Institute of Medical Education 1998;32:1-4

Mandeep Dhillon, Senior Consultant Orthopaedic Surgeon, Apollo Hospitals, Colombo Sri Lanka, and Harpreet S Gill, Consultant Orthopaedic Surgeon, Primary Health Center, Model Town, Patiala, Punjab, India. Correspondence: MD, e-mail <mandeep@drdhillon.net> (Competing interests: none declared).

\section{Experts are dangerous}

I am an expert of electricity. My father occupied the chair of applied electricity at the state prison.

WC Fields, (commenting on the value of “experts.” Editor, $C M J$ ) 